\title{
THE ACUTE EFFECTS OF WARM-UP, STATIC AND DYNAMIC STRETCHING EXERCISES ON BICEPS BRACHII MUSCLE FUNCTION IN FEMALE BASKETBALL PLAYERS
}

\author{
Marju Veevo, Jaan Ereline, Eva-Maria Riso, Helena Gapeyeva, \\ Mati PäÄSUKe \\ Institute of Exercise Biology and Physiotherapy, University of Tartu, Tartu, Estonia
}

\begin{abstract}
Static and dynamic stretching exercises are often used in sports before competition and training sessions. Recent studies have shown different effects of dynamic and static stretching exercises on physical performance capacity. The aim of the present study was to evaluate the acute effect of warm-up, 30 s static and dynamic stretching exercises on biceps brachii muscle function. Nine athletes were examined twice in different days: after performing static and dynamic stretching exercise. Isometric maximal voluntary contraction characteristics were measured using dynamometry; muscle tone, elasticity and stiffness characteristics of biceps brachii muscle were measured using myotonometer Myoton-3. All measurements were performed before and after the warm-up (5-min jogging with self-selected speed), immediately after stretching exercise and after $10 \mathrm{~min}$ rest. Warm-up did not cause any changes in the isometric maximal voluntary contraction, strength of elbow flexor muscle as well as in muscle tone and elasticity. Static stretching was effective to increase elasticity of biceps brachii muscle, but did not influence the isometric strength characteristics of elbow flexor muscles. Shortening of isometric contraction latency of elbow flexor muscles after dynamic stretching exercise and increase of strength after $10 \mathrm{~min}$ rest were noted. Therefore this type of stretching can be recommended to prepare muscles for explosive maximal exercise.
\end{abstract}

Keywords: static stretching exercises, dynamic stretching exercises, isometric strength, muscle stiffness, muscle tone 


\section{INTRODUCTION}

Warm-up is an essential part of sports activities helping to prepare the organism for the following exertions. Athletes use many different types of stretching that are usually based only on their personal preference, but no optimal type or amount of stretching exercises has been identified [6]. The intended purposes of stretching before an athletic event are to insure that the individual has sufficient flexibility of joints to perform the athletic activity optimally and to decrease muscle stiffness or increase muscle compliance, thereby theoretically decreasing injury risk [9]. In addition to aerobic warm-up, different kinds of stretching exercises are widely used, including ballistic, proprioceptive neuromuscular facilitation, static and dynamic stretching $[6,9]$. To find out the optimal warm-up program and to select exercises which improve the contraction speed and power of muscle-tendon unit, and help to prevent injuries simultaneously are actual in sports practise. The studies of recent 10 years have not found which kind of stretching is more effective for warm-up - the static or dynamic stretching exercises. Static stretching exercises increase the joint range of motion almost two-fold as compared to dynamic stretching exercises but they have no positive effect on the muscle contraction characteristics and explosive muscle strength $[1,4,10]$. It has been also demonstrated that warm-up which includes dynamic stretching exercises is effective to prepare the muscle for performance during high-speed movements [5]. In many sports events, it is important to achieve the optimal condition of muscles in very short time, especially among the exchange players in ball games or in throwing events between the trials. In most previous studies, the function of lower limb muscles has been determined, but in several sports events the maximal exertion of upper limbs is very important. The aim of this study was to analyse the acute effect of warm-up and compare the influence of static and dynamic stretching exercises on the biceps brachii (BBr) muscle function in female basketball players.

\section{MATERIALS AND METHODS}

\section{Participants}

Nine healthy adult female basketball players (mean age \pm SE: $22.0 \pm 0.8$ years, body height $1.71 \pm 0.03 \mathrm{~m}$, body weight $67.9 \pm 2.34 \mathrm{~kg}$ ) participated voluntarily in this study. Subjects had been trained for $13.6 \pm 0.9$ years with a mean load $11.0 \pm 1.53 \mathrm{hrs}$ per week and competed at national level. Before the beginning of the testing session, all subjects completed an informed consent form. The study carried the approval of the Ethics Committee of the University of Tartu. 


\section{Design of experiment}

The subjects were evaluated twice after day of rest from training. The period between two examinations was 1-5 weeks. Each examination included two stages. The first stage was similar in both examinations: the subjects performed a warm-up ( 5 min jogging with individually chosen speed) after first measurements. Next 2-3 min after warm-up measurements was performed. The second stage consisted of pain-free stretching exercise for the biceps brachii muscle-tendon unit: in first examination $30 \mathrm{~s}$ of passive stretching, and in the other examination accordingly $32 \mathrm{~s}$ of active dynamic stretching (8 times 4-s stretching exercises). Measurements were performed immediately after the stretching and fourth measurements after 10-min rest (Figure 1).

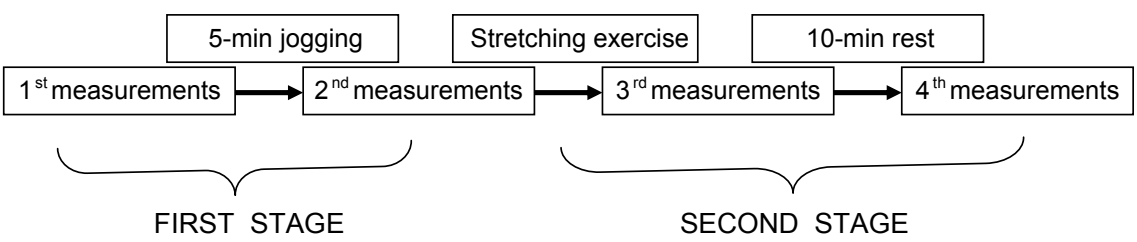

Figure 1. Design of the experiment. After first measurements the subjects jogged for 5 -min and $2^{\text {nd }}$ measurements were carried out. After $2^{\text {nd }}$ measurements the subjects performed stretching exercise, followed by $3^{\text {rd }}$ measurements. Then the subjects rested for $10 \mathrm{~min}$, and finally, $4^{\text {th }}$ measurements were performed.

\section{Isometric strength measurement}

The isometric maximal voluntary contraction (MVC) force of biceps brachii was investigated using a custom made chair and standard dynamometer. The subjects were required to perform a maximal contraction of elbow flexor muscle beginning from the switch of signal lamp on and to held the maximal exertion as hard as possible during $2 \mathrm{~s}$ and to relax quickly when the signal disappeared. The following characteristics of isometric strength were recorded: isometric maximal voluntary contraction force (Fmax, N), rate of peak force development (RFD) $0.2 \mathrm{~s}$ after the beginning of contraction, RFD at the level of $50 \%$ of MVC and the latency of contraction (LATc).

\section{Muscle tone measurement}

The stiffness $(\mathrm{N} / \mathrm{m})$ of biceps brachii muscle at rest was registered using myotonometer Myoton 3 (Müomeetria OÜ, Estonia) in sitting position of the subject in abovementioned chair. In present study the stiffness of the muscle is accepted as the property to resist the forces trying to change its shape [11]. To 
compare the relative changes, the results of first measurement on both examination days were accepted as 100\% (baseline level).

\section{Statistical analysis}

Data are presented as means and standard errors of means. The Student paired $t$-test was used for comparison of groups. A level of $\mathrm{p}<0.05$ was selected to indicate statistical significance. The data of first measurement on the both examinations were used as baseline level.

\section{RESULTS}

\section{Changes of muscle stiffness}

The changes in muscle stiffness are demonstrated in Figure 2. No significant difference in biceps brachii muscle stiffness was noted after warm-up on either examination day as compared to baseline level. Biceps brachii muscle stiffness decreased significantly $(\mathrm{p}<0.001)$ after static stretching exercise as compared to baseline level and increased after 10 -min rest $(\mathrm{p}<0.001)$. Biceps brachii stiffness decreased also after dynamic stretching exercise but these changes did not differ significantly $(\mathrm{p}>0.05)$.

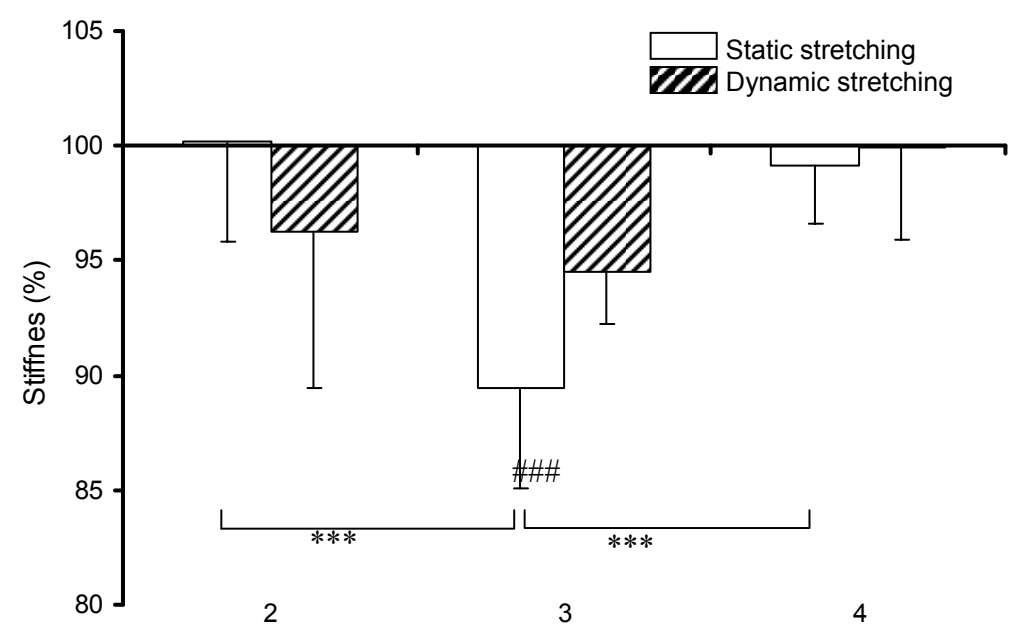

Figure 2. The changes in muscle relative stiffness after static and dynamic stretching. ${ }^{* * \star} p<0.001, \# \# \# p<0.001$. 


\section{Changes in strength characteristics}

No significant changes in isometric maximal voluntary contraction force (MVC) were registered on either day of examinations (Figure 3A). No significant changes were found in majority of parameters of contraction force in both examinations. The significant increase of RFD 0.2 after BOTH stretching exercise $(\mathrm{p}<0.05)$ was found (Figure $3 \mathrm{~B})$. $\mathrm{RFD}_{50 \%}$ did not differ significantly on either examination day. After warm-up and static stretching exercise LATc did not decrease significantly. Dynamic stretching exercise caused the significant shortening of LATc as compared to baseline level $(\mathrm{p}<0.05)$ and to warm-up $(\mathrm{p}<0.01)$. After the 10 -min rest LATc prolonged significantly as compared to after stretching $(\mathrm{p}<0.01)$ (Figure 4$)$.
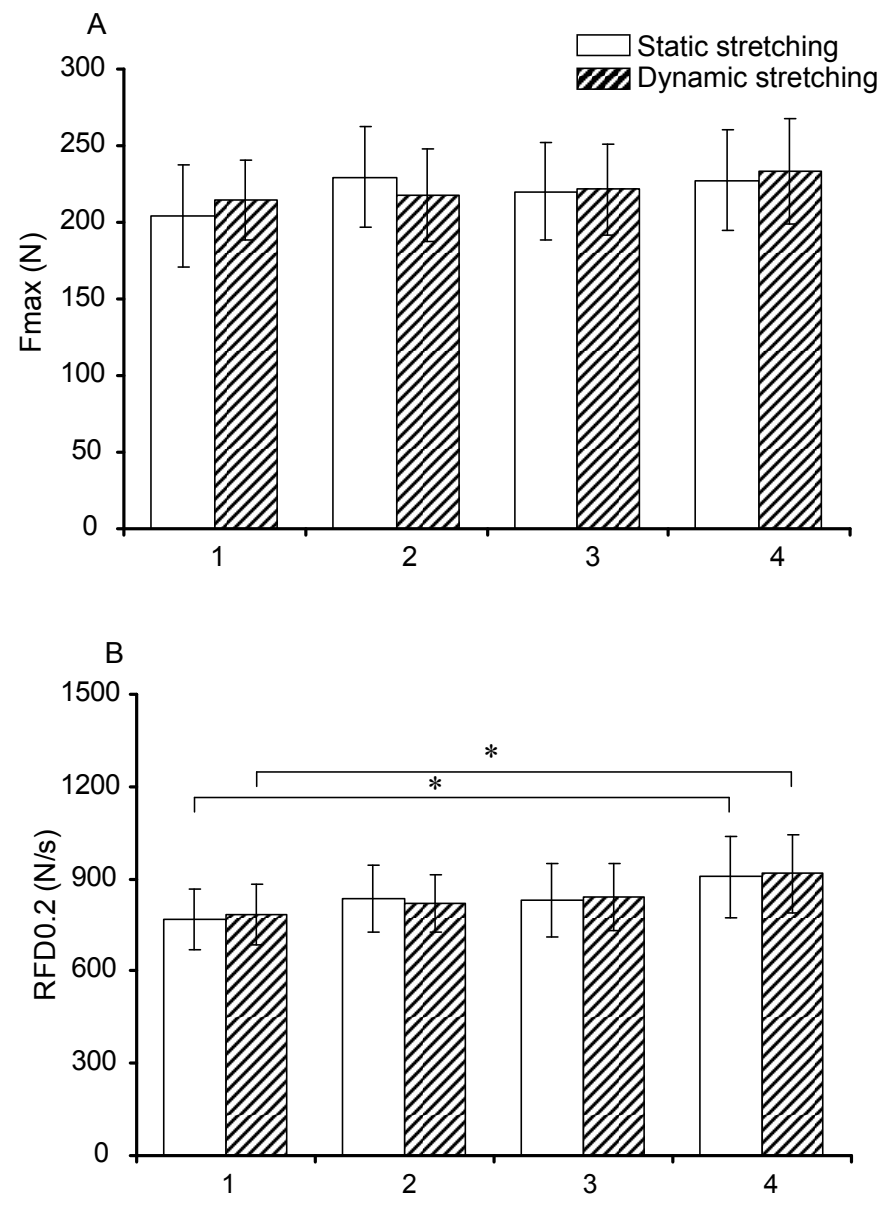

Figure 3. A: The changes in isometric maximal voluntary contraction force (mean \pm SE). B: The changes in RFD0.2 (means \pm SE). ${ }^{*} p<0.05$ 


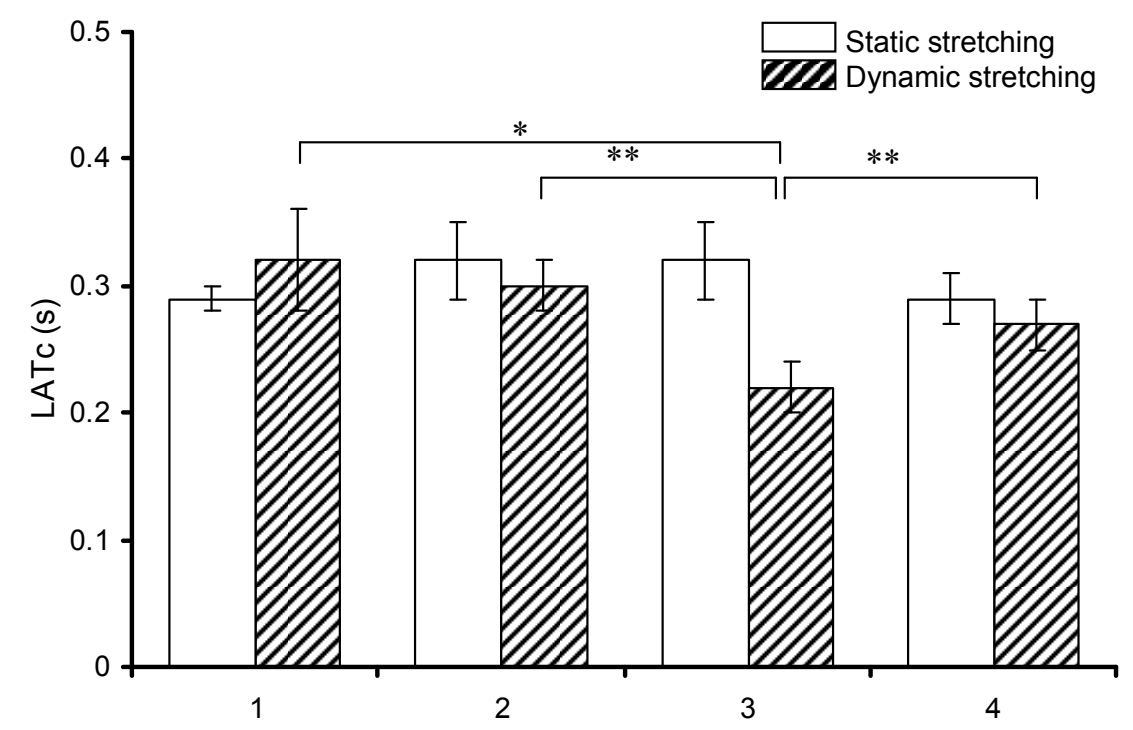

Figure 4. The changes in latency of contraction after static and dynamic stretching (LATc) (mean \pm SE). ${ }^{*} p<0.05,{ }^{\star \star} p<0.001$.

\section{DISCUSSION}

In the present study, a comparison of the acute effect of different type of stretching exercises on the function of biceps brachii muscle in female basketball players was performed. The main finding of our study was that the muscle tone characteristics, expressed as muscle stiffness, decreased significantly after static stretching exercise but not after dynamic stretching exercise. Neither static nor dynamic stretching exercise had significant influence on isometric maximal voluntary contraction force generation. Latency of MVC shortened significantly after dynamic exercise as compared to baseline level. Several authors confirm that warm-up helps to achieve the quicker spread of the nerve impulse [5]. Pearce et al. [7] demonstrate that the improvement of neuromuscular electrical conduction has been caused by the better electrical conduction of contractile apparatus and muscle fibers. The electrical conduction increases during warm-up in both active and passive skeletal muscles [7]. Our results are not in full accordance with abovementioned findings, because we did not find significant changes of LATc immediately after stretching on both examination days.

The stiffness of biceps brachii muscle decreased after $30 \mathrm{~s}$ of static stretching but after 10-min rest did not differ from the baseline level. This finding can be explained by mechanical theory which states that impairment of muscle 
stiffness leads to the decrease of force, because the optimal distance between actin and myosin filaments is disturbed causing changes in force production [3]. We did not find the decrease in force production accompanied by a decrease of stiffness in our experiment. In contrary to our results, previous study did not demonstrate changes in muscle stiffness after static stretching exercise [2]. The duration of stretching exercise can be the reason of different results among these studies. In many experiments, the repeated series of static stretching have been used and therefore the duration of static exercise lengthens. The results of Yamaguchi and Ishii [12] are in accordance with ours: $30 \mathrm{~s}$ static stretching had no significant effect on the force parameters in their study [12].

It is well known that dynamic stretching increases the intramuscular temperature [8]. We did not measure the intramuscular temperature in our experiment but it can be assumed that increased temperature could cause the shortening of LATc [8]. LATc decreased significantly after dynamic stretching exercise - its values were $31 \%$ lower than baseline level and $27 \%$ lower than after warm-up. After $10 \mathrm{~min}$ rest LATc was recovered to the control level $(\mathrm{p}<0.01)$. Many sports events require movements including dynamic stretching and therefore it can be supposed that using of dynamic stretching in training could increase the effectiveness of movements [5]. As the co-effect of warm-up program and rest, $\mathrm{RFD}_{0.2}$ increased after $\mathrm{BOTH}$ stretching exercise. This can be explained with the cumulative effect of all manipulations.

We can conclude that $5 \mathrm{~min}$ warm-up and following static stretching exercise of biceps brachii muscle decrease the muscle stiffness for $10 \mathrm{~min}$ more as compared to dynamic stretching exercise. At the same time, the force generation capacity of elbow flexor muscles and the latence time of contraction generation capacity did not change significantly. The latence time of muscle contraction decreased as a result of warm-up and following dynamic stretching. Explosive strength of elbow flexor muscles increased significantly after warm-up and dynamic stretching as well as after $10 \mathrm{~min}$ rest which demonstrates the positive co-effect of these activities on the contractile capacity of skeletal muscle. The dynamic stretching exercises can be recommended to prepare the muscles for maximal exertions.

\section{REFERENCES}

1. Bandy WD, Irion JM, Briggler M. (1998) The effect of static stretch and dynamic range of motion training on the flexibility of the hamstring muscles. J Orthop Sports Phys Therapy, 27: 295-300 
2. Cannavan D, Coleman DR, Blazevich AJ. (2012) Lack of effect of moderate-duration static stretching on plantar flexor force production and series compliance. Clin Biomech, 27: 306-312

3. Evetovich TK, Nauman NJ, Conley DC, Todd JB. (2003) Effect of static stretching of the biceps brachii on torque, electromyography, and mechanomyography during concentric isokinetic muscle actions. J Strength Cond Res, 17: 484-488

4. Kistler B, Walsh MS, Horn T, Cox R. (2010) The acute effects of static stretching on the sprint performance of collegiate men in the 60 - and $100 \mathrm{~m}$ dash after a dynamic warm-up. J Strength Cond Res, 24: 2280-2284

5. Little T, Williams AG. (2006) Effects of differential stretching protocols during warm-ups on high-speed motor capacities in professional soccer players. J Strength Cond Res, 20: 203-207

6. McHugh MP, Cosgrave CH. (2010) To stretch or not to stretch: the role of stretching in injury prevention and performance. Scand J Med Sci Sports, 20: 169-181.

7. Pearce A, Rowe GS, Douglas GW. (2012) Neural conduction and excitability following stretching. J Sci Med Sport, 15: 164-168

8. Pearce AJ, Kidgell DJ, Zois J, Carlson JS. (2009) Effects of secondary warm-up following stretching. Eur J Sport Sci, 105: 175-183

9. Sekir U, Arabaci R, Akova B, Kadagan SM. (2010) Acute effect of static and dynamic stretching on leg flexor and extensor isokinetic strength in elite women athletes. Scand J Med Sci Sports, 20: 268-281

10. Unick J, Jieffer HS, Cheesman W, Feeney A. (2005) The acute effects of static and ballistic stretching on vertical jump performance in trained woman. J Strength Cond Res, 19: 206-212

11. Vain A. (1997) Method and device for recording mechanical oscillations in soft biological tissues. European Patent. No 0897287

12. Yamaguchi T, Ishii K. (2005). Effects of static stretching for 30 seconds and dynamic stretching on leg extension power. J Strength Cond Res, 19: 677-683

\section{Correspondence to:}

Marju Veevo

Institute of Exercise Biology and Physiotherapy

Faculty of Exercise and Sport Sciences

University of Tartu

E-mail: v.marju@gmail.com

Telephone: (+372) 7376286

Fax: $(+372) 7376286$ 